\title{
The effect of prolonged formalin fixation on the staining characteristics of archival human brain tissue
}

\author{
A. Alrafiah ${ }^{1,3}$, R. Alshali2, 3 \\ ${ }^{1}$ Department of Laboratory Technology, Faculty of Applied Medical Sciences, King Abdulaziz University, Jeddah, Saudi Arabia \\ ${ }^{2}$ Department of Anatomy, Faculty of Medicine, King Abdulaziz University, Jeddah, Saudi Arabia \\ ${ }^{3}$ Neuroscience Research Unit, Faculty of Medicine, King Abdulaziz University, Jeddah, Saudi Arabia
}

[Received: 16 August 2018; Accepted: 3 October 2018]

Background: Neurodegenerative disorders include wide range of conditions, which affect millions of people worldwide. Unfortunately, they are incurable and irreversibly progressive. Immunohistochemical staining of paraffin-fixed tissues for both diagnostic and research purposes are widely used. However, large amount of brain tissues are fixed but little is known about whether they are suitable for retrospective studies. The study aimed at investigating the effects of prolonged formalin fixation time on immunohistochemical expression of some common neurodegenerative markers in archival brain specimens. Materials and methods: Twenty brain specimens were obtained from human cadavers in the Anatomy Department of King Abdulaziz University that were prefixed in 10\% formalin. They were divided into two equal groups according to time of fixation: group 1 - less than 1 year, group 2 - up to 20 years. Histological examination of white and grey matter was done using haematoxylin and eosin, luxol fast blue (LFB) for myelin staining, Congo red for amyloid plaques, CD 68 for microglial cells, tenascin-C (large extracellular matrix glycoprotein) and caspase 3 antibody for apoptotic cells. Results: For both groups, corpus callosum sections displayed myelination with LFB staining. The distribution of CD 68 positive microglial cells was evident in frontal and temporal grey matter, but not in corpus callosum sections. Strongly positive masses were seen in Congo red-stained frontal and temporal sections. Anti-caspase 3 immunostaining revealed positively stained neurons. Conclusions: Histological and immunohistochemical techniques yielded reproducible staining results when applied to human brain tissue stored in formalin for long periods; so they can be used in well preserved biobank material which are the most targeting research areas in neuropathology. (Folia Morphol 2019; 78, 2: 230-236)

Key words: human brain, immunohistochemistry, formalin fixed, neurodegenerative markers

Address for correspondence: Dr. A. Alrafiah, Department of Laboratory Technology, Faculty of Applied Medical Sciences, King Abdulaziz University, P.O. Box 80200, Jeddah 21589, Saudi Arabia, tel: +966 (01) 26401000, ext. 23495, fax: +966 (01) 26401000, ext. 21686, e-mail: aalrafiah@kau.edu.sa 


\section{INTRODUCTION}

Paraformaldehyde (PFA) is the most commonly used fixative material that preserves the gross anatomy of organs and the histological details of tissue compared to other fixatives like acetone and alcohol. For formalin fixation process, tissue is soaked in $10 \%$ neutral buffered formalin for 24-48 $\mathrm{h}$ [8].

Formalin fixation is a chemical process in which formaldehyde forms covalent bonds and produces cross-links. Thus, this fixation may limit antibodies permeation into the tissue, decrease the accessibility of antigenic determinants and alter the structure of the epitopes to be visualised by immunohistochemistry (IHC) $[16,20]$. Moreover, prolonged time of formalin fixation can modify the structure of the epitope to be visualised by IHC [16] and hence, reduce the antigenicity and efficiency of IHC [20]. Despite that, researches up to date revealed that some antigens are sensitive to the fixation time more than others $[4,16,31]$. However, only few studies showed that human postmortem brain tissue can be fixed for long periods up to several decades, and that some epitopes could be preserved for years before declining in their immunoreactivity [7]. In contrast, other studies indicated that prolonged fixation may affect the efficiency of IHC [1].

Neurodegenerative diseases (NDDs) refer to a wide range of conditions in which the neurons in the brain and spinal cord become injured, degenerated or eventually die. Those neuron cells actually cannot regenerate themselves nor be replaced by the body.

Nowadays, NDDs became more common, especially Parkinson's, Alzheimer's and multiple sclerosis. Unfortunately, they are incurable and irreversibly progressive conditions that lead to hopeless situations starting from mental malfunctioning (dementias) to more serious movement impairments (ataxias). Those NDDs have some common markers such as CD 68, tenascin- $C$ and caspase 3 that can be identified by IHC. Also, luxol fast blue (LFB) and Congo red (CR) are specific stains confirming degenerative changes.

CD 68 is a useful marker for microglia, which are specialised macrophage cells in the brain [11]. Microglia are activated and become phagocytes in response to central nervous system (CNS) infection or damage [24], which occurs in multiple sclerosis, Alzheimer's disease and Parkinson's disease. Tenascin- $\mathrm{C}$ is expressed transiently by astrocytes during embryonic development of the CNS [29]. Fibroblasts and other tissues express it during cell differentia- tion and repair [18]. Tenascin-C is also considered a potential biomarker induced during inflammation or cancer [17]. Caspase 3 is essential for normal brain development and cell apoptosis [21]. LFB stain, also known as (myelin stain), is commonly used to detect demyelination in the CNS $[5,12]$. CR is the diagnostic dye for amyloidosis, which is the hallmark of degenerative diseases such as Alzheimer's disease [6]. The CR staining is a reliable technique to detect amyloid deposits in tissues, especially if it is confused with other materials when stained by haematoxylin and eosin (H\&E).

Our aim in this study is to determine the effect of prolonged formalin fixation on immunohistochemical expression of a panel of common neurodegenerative markers in archival human brain specimens. The study of postmortem human brain tissue is a powerful method to investigate various neurological diseases, thus providing better insight into their pathogenesis.

\section{MATERIALS AND METHODS}

Twenty brain specimens were obtained from human cadavers in the Anatomy Department of King Abdulaziz University that were prefixed in $10 \%$ formalin. They were divided into two equal groups according to time of fixation: group 1 - less than 1 year, group 2 - up to 20 years.

Five-micrometre $(4 \mu \mathrm{m})$ sections of formalin-fixed, paraffin-embedded (FFPE) tissue from the frontal and temporal gyrus region were processed and sectioned.

Histological examination of white and grey matter was done using H\&E, LFB for myelin staining, CR for amyloid plaques, and IHC was performed to assess the expression of common molecular markers for neurodegenerative diseases using antibodies against CD 68 for microglial cells, tenascin-C (large extracellular matrix glycoprotein) and caspase 3 antibody for apoptotic cells (Table 1).

Immunohistochemistry was performed using fully automated Leica Bond machine. Epitope retrieval was performed in all samples using Leica ER2 solution (AR9640) at $97^{\circ} \mathrm{C}$. Sections were placed in $\mathrm{H}_{2} \mathrm{O}_{2}(3 \%)$ for $30 \mathrm{~min}$ in methanol.

\section{RESULTS}

Histological examination of H\&E-stained sections of frontal and temporal grey matter and corpus callosum revealed normal appearance. Examination of LFB-stained corpus callosum sections of both groups revealed darkly stained blue myelinated areas in some 
Table 1. Antibodies and conditions

\begin{tabular}{lcccc}
\hline Antibody & Species & Clone/company & Dilution & Antigen retrieval technique \\
\hline Anti-tenascin-C & Mouse & Polyclonal/Merck Millipore (AB19013) & $1: 200$ & Automated ER1 \\
Anti-CD 68 & Mouse & Clone kp1/Dako & Prediluted & Automated ER2 \\
Caspase 3 & Rabbit & Ab2302 & $1: 100$ & Automated ER1 \\
\hline
\end{tabular}
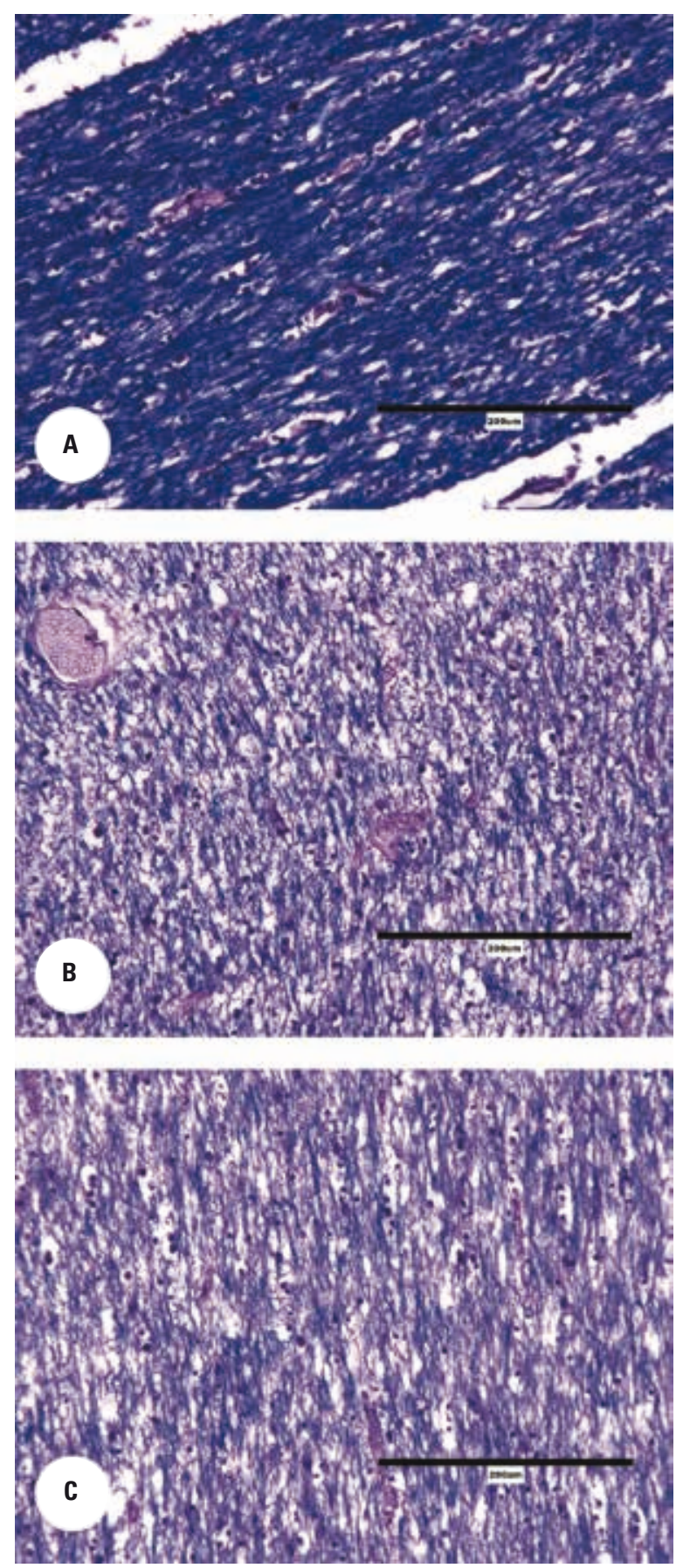

Figure 1. Luxol fast blue stained - corpus callosum sections; A. Group 1, showing dark blue areas of myelination; B. Group 1, showing pale blue areas denoting hypomyelination; C. Group 2, showing areas of hypomyelination. sections whereas hypo-myelinated sections showed pale blue staining (Fig. $1 \mathrm{~A}-\mathrm{C}$ ).

In CD 68-immunostained sections of both studied groups, positively stained microglia cells were seen in frontal and temporal grey matter sections (Fig. 2A, B, respectively) while in corpus callosum sections no positive immunostaining was detected (Fig. 2C).

Examination of CR-stained sections of frontal and temporal grey matter of both groups revealed dull red staining of the neurons as well as the neuropil (Fig. 3A). Strongly positive congophilic masses of variable sizes were also seen among neurons (Fig. 3B, 3C). In some sections, the blood vessels revealed amorphous congophilic amyloid deposits in their walls (Fig. 3D).

In tenascin-C-stained sections, differences in the staining properties were observed among studied groups. In corpus callosum sections, there was positive staining of the extra cellular matrix in both groups (Fig. 4A, B), while in frontal and temporal grey matter sections positive intracytoplasmic as well as extracellular staining were seen (Fig. 4C, D).

Frontal and temporal grey matter sections of both groups that were stained with anti-caspase 3 antibodies showed multiple positively stained neurons as well as some positively stained endothelial cells lining blood vessels (Fig. 5A, B). The caspase 3-positive immunostaining was seen to be cytoplasmic (Fig. 5C).

\section{DISCUSSION}

In this work, we evaluate the effect of prolonged formalin fixation on the efficiency of IHC staining of archival human brain specimens. This approach has been put to most practical use through the use of specific antibodies raised against these 'molecular markers'. Several biomarkers and stains for neurodegenerative diseases were used.

Luxol fast blue stain was used to assess the feasibility of myelin staining in archival sections. Our results were similar to those of Sheaffer et al. [22] who examined brain sections, which were fixed in formalin for up to 53 years, for myelin loss and astrocytosis with satisfactory results. Others studied the relation 

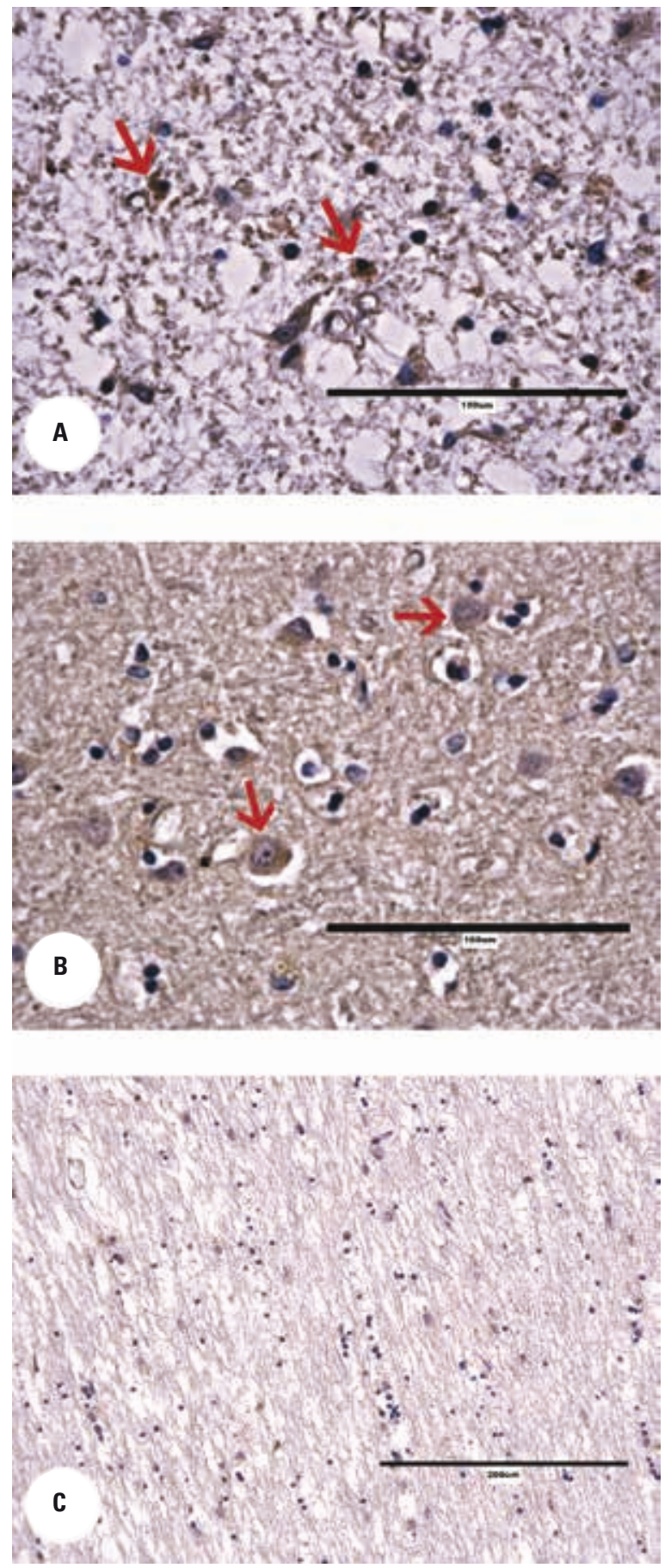

Figure 2. CD 68 immunostaining; A. Frontal lobe section of group 1, showing positively stained microglial cells (arrows); B. Temporal lobe section of group 2, showing positively stained microglial cells (arrows); C. Corpus callosum section of group 1, showing negative immunostaining for microglial cells.
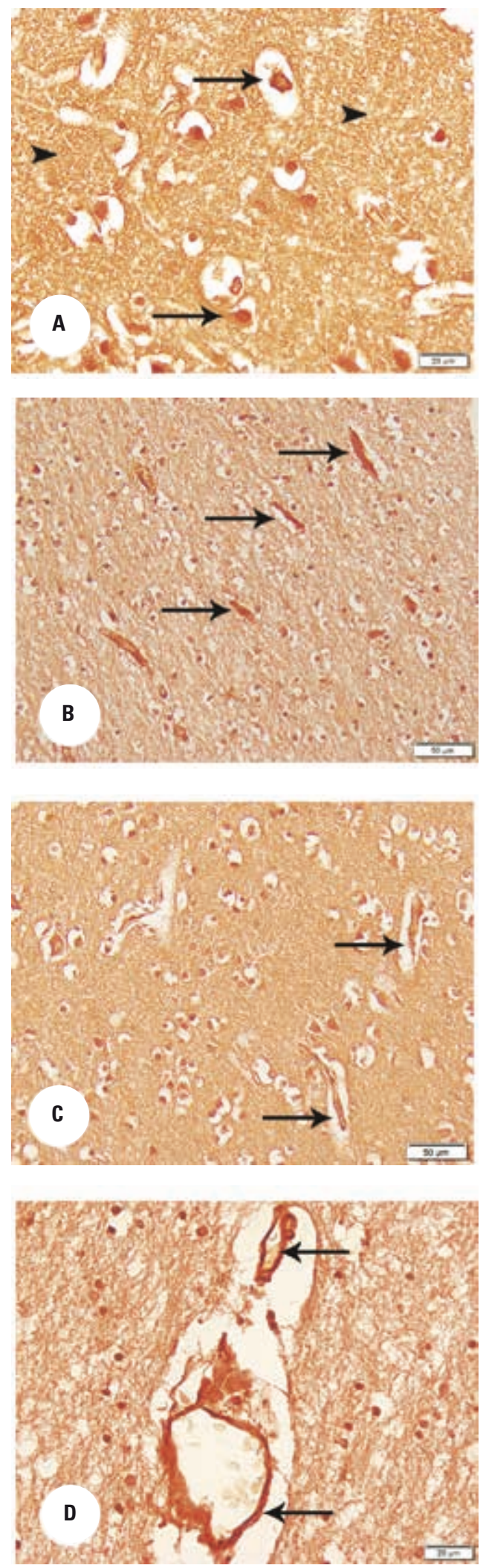

Figure 3. Congo red stained - frontal lobe sections; A. Group 1, showing dull red staining of neurons (arrows) as well as neuropil (arrowhead); B. Group 2, showing some small positively stained congophilic masses (arrows); C. Group 1, showing large positively congophilic masses (arrows) among positively stained neurons; D. Group 2, showing congophilic deposits (arrows) within the wall of some blood vessels. 

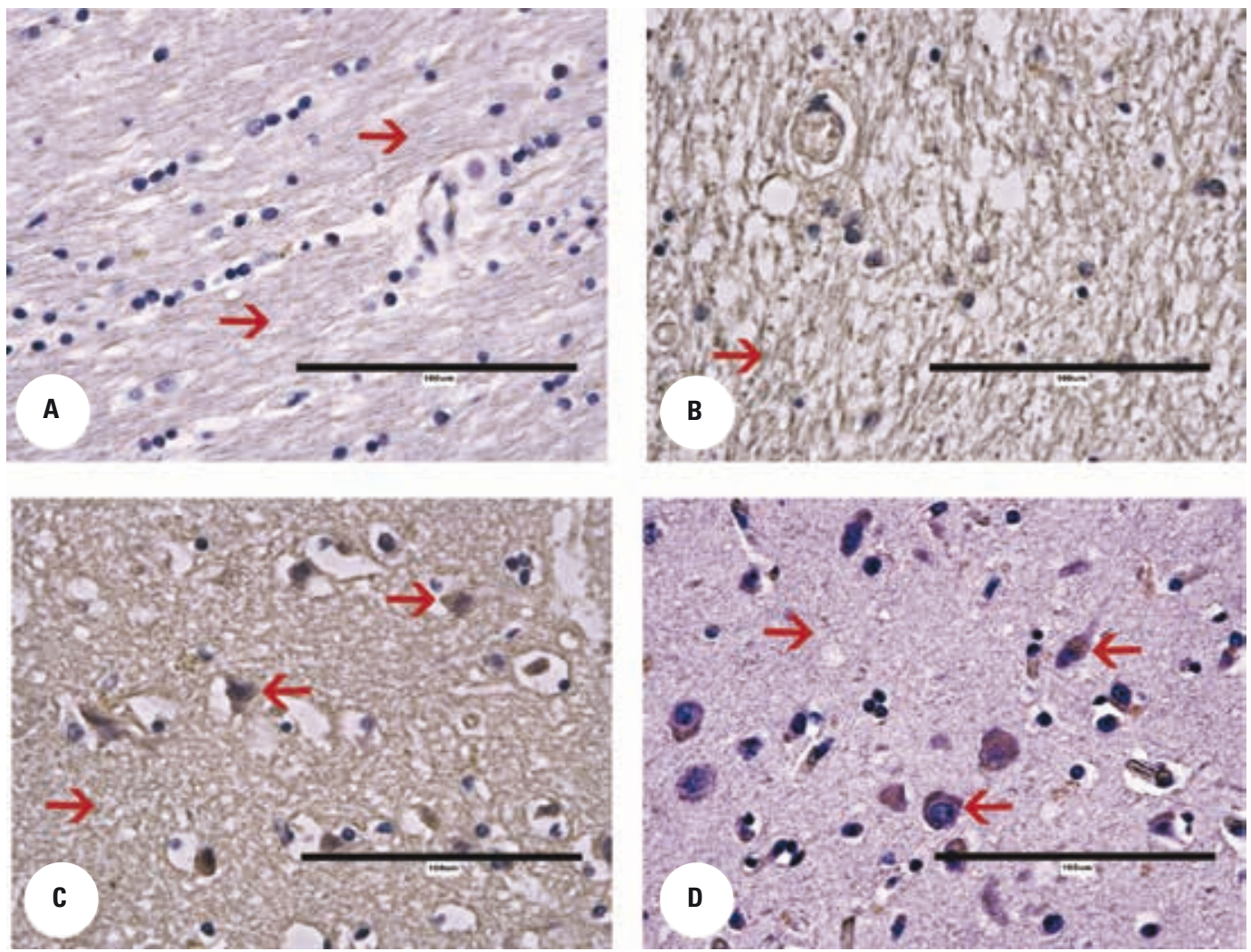

Figure 4. Tenascin-C immunostaining; A. Corpus callosum section of group 1, showing positive staining for tenascin- $\mathrm{C}$ in the extra cellular matrix (arrowhead); B. Corpus callosum section of group 2, showing positive staining for tenascin-C in the extra cellular matrix (arrowhead); C. Frontal grey matter section of group 2, showing positive staining for tenascin-C intracytoplasmic (arrow) as well as in the extra cellular matrix (arrowhead); D. Temporal grey matter section of group 1, showing positive staining for tenascin-C intracytoplasmic as well as in the extra cellular matrix (arrowhead).

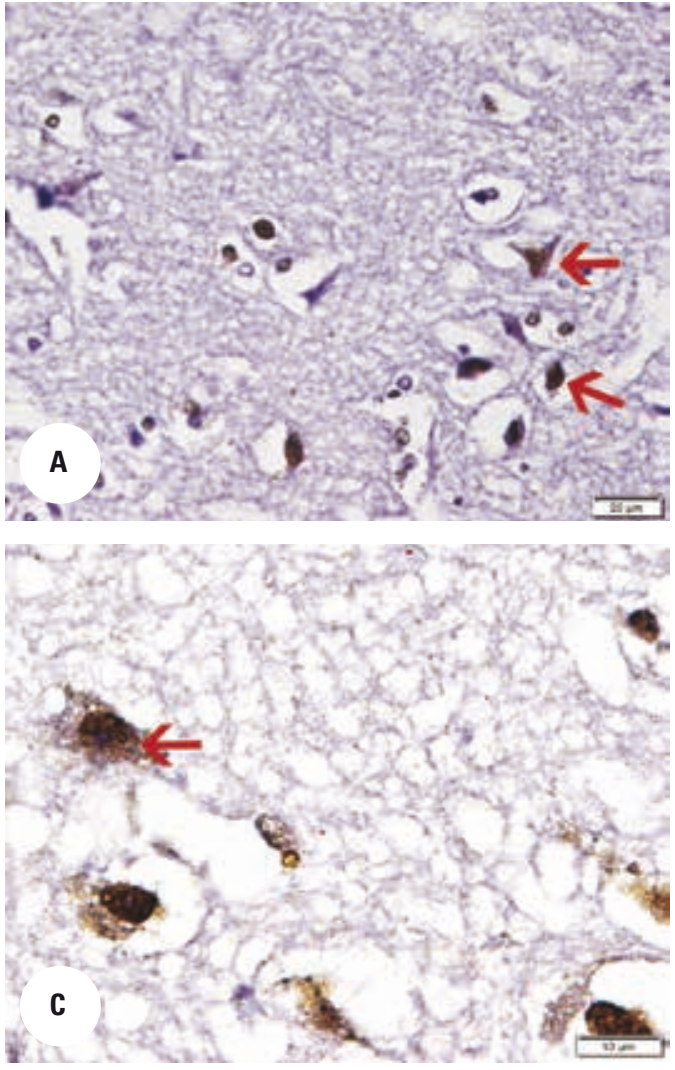

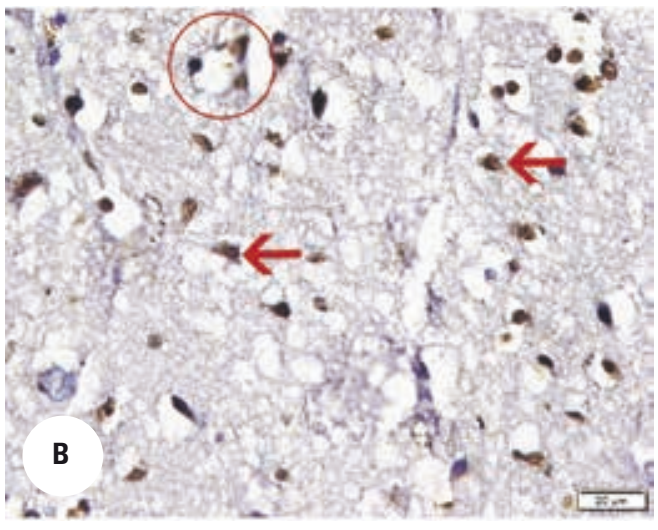

Figure 5. Anti-caspase 3 immunostaining of frontal grey matter section; A. Group 1, showing some caspase 3 positive neurons (arrows); B. Group 2, showing caspase 3 positive immunostained neurons (arrows) as well as in some endothelial lining of a blood vessel (encircled); C. Group 2, showing cytoplasmic positive caspase 3 immunostaining (arrow). 
between brain stiffness and myelin content in brain sections stained with LFB and quantified using image analyser. They stated that brain stiffness increases with myelin content [28].

According to Walker and Lue [26], microglia can be detected in human brain sections, particularly in diseased brains, using CD 68-antibody. This may be attributed to changes in the properties of microglia and can be considered as a common feature of human neurodegenerative diseases [26]. That was consistent with our results of $C D 68$ immunostaining to detect microglia in studied sections.

Ando et al. [3] found that during life, the accuracy of amyloid deposition in brain by clinical diagnosis is only $65 \%$; however, definite diagnosis is reached by examination of the brain at autopsy. Previous work showed that the detection of condensed beta-amyloid in a secondary structure in brain parenchyma (amyloid plaques) and blood vessels can be confirmed using CR stain [30]. In our studied sections, CR staining successfully detected amyloid deposits in the wall of blood vessels, and in support to our data, Smith [23] stated that cerebral amyloid angiopathy is considered a morphological characteristic of Alzheimer disease. However, it may be also seen in the brains of elderly people who don't have any neurological problems.

The extracellular matrix glycoprotein tenascin- $\mathrm{C}$ is recognised in embryogenesis and wound healing as well as in some neoplasia, such as gliomas, where it can be used as prognostic marker for tumour recurrence [10]. The immunostained sections in this study revealed varied staining pattern similar to results of a study comparing the expression of tenascin- $\mathrm{C}$ in grey and white matter of control human brains; significant differences was found by using Western blot analysis or IHC [14]. Lee and McKinnon [13] stated that apoptosis occurring in the nervous system may be seen either during normal development, or can be induced by disease or DNA damage.

It has been stated that caspase 3 protein is essential in mediating cell apoptosis, because it is affected by the cell surface death receptor [25]. On this basis, our studied sections showed variable caspase 3 positive immunostaining. In support to our figures, Machaalani et al. [15] studied the effect of tissue fixation on caspase 3 immunostaining in brain tissue. They found variations in the efficiency of IHC staining using commonly available antibodies for caspase 3, which was dependent on the animal species and tissue fixation method.
Our results point to the preservation of the antigenicity of some epitopes in archival human brain tissue prefixed in formalin for long time. In agreement with our results, Webster et al. [27] revealed negligible effects of prolonged time of formalin fixation on antigen detection for many used antibodies in IHC staining in domestic animals. All results collectively encourage the use of $\mathrm{IHC}$ as a tool in diagnostic pathology [19].

According to another study, exogenous factors such as premortem or post mortem conditions can affect postmortem brain tissue preservation [2]. The question asked by Grillo et al. [9] is whether IHC on archival formalin-fixed paraffin blocks has an expiry date? Grillo et al. [9] studied the factors affecting immunoreactivity in paraffin embedded sections after long term storage of formalin fixation. They stated that loss of antigenicity was relational to the age of tissue section and mode of storage. The cold storage has the minimum effect and heat pretreatment for antigen retrieval was needed for all antigens.

\section{CONCLUSIONS}

It is concluded that long-term formalin fixation of human brains does not affect their antigenicity. Therefore there is increasing demand for brain banks with proper selection and collection of postmortem brain tissue. Information on individual's previous neurological condition must be also provided. This represents a unique archive for scientific research worldwide using different histological (histochemistry and IHC) techniques to study neurodegenerative diseases.

\section{Acknowledgements}

Neuroscience research unit at King Abdulaziz University, Jeddah, Saudi Arabia.

\section{REFERENCES}

1. Alafuzoff I, Pikkarainen M, Al-Sarraj S, et al. Interlaboratory comparison of assessments of Alzheimer disease-related lesions: a study of the BrainNet Europe Consortium. J Neuropathol Exp Neurol. 2006; 65(8): 740-757, doi: 10.1097/01. jnen.0000229986.17548.27, indexed in Pubmed: 16896308.

2. Alturkistani HA, Tashkandi FM, Mohammedsaleh ZM. Histological Stains: A Literature Review and Case Study. Glob J Health Sci. 2015; 8(3): 72-79, doi: 10.5539/gjhs. v8n3p72, indexed in Pubmed: 26493433.

3. Ando Y, Haraoka K, Terazaki H, et al. A novel tool for detecting amyloid deposits in systemic amyloidosis in vitro and in vivo. Lab Invest. 2003; 83(12): 1751-1759, doi: 10.1097/01.lab.0000101701.87433.c5.

4. Arber D. Effect of prolonged formalin fixation on the immunohistochemical reactivity of breast markers. Appl 
Immunohistochem Mol Morphol. 2002; 10(2): 183-186, doi: 10.1097/00129039-200206000-00015.

5. Carriel V, Campos A, Alaminos M, et al. Staining Methods for Normal and Regenerative Myelin in the Nervous System. Methods Mol Biol. 2017; 1560: 207-218, doi: 10.1007/978-1-4939-6788-9 15, indexed in Pubmed: 28155156.

6. Clement CG, Truong LD. An evaluation of congo red fluorescence for the diagnosis of amyloidosis. Hum Pathol. 2014; 45(8): 1766-1772.

7. Evers $P$, Uylings $H$. An optimal antigen retrieval method suitable for different antibodies on human brain tissue stored for several years in formaldehyde fixative. J Neurosci Methods. 1997; 72(2): 197-207, doi: 10.1016/ s0165-0270(96)02204-2.

8. Fox $\mathrm{CH}$, Johnson FB, Whiting J, et al. Formaldehyde fixation. J Histochem Cytochem. 1985; 33(8): 845-853, doi: 10.1177/33.8.3894502, indexed in Pubmed: 3894502.

9. Grillo F, Pigozzi S, Ceriolo P, et al. Factors affecting immunoreactivity in long-term storage of formalin-fixed paraffin-embedded tissue sections. Histochem Cell Biol. 2015; 144(1): 93-99, doi: 10.1007/s00418-015-1316-4, indexed in Pubmed: 25757745.

10. Herold-Mende C, Mueller MM, Bonsanto MM, et al. Clinical impact and functional aspects of tenascin- $C$ expression during glioma progression. Int J Cancer. 2002; 98(3): 362-369, indexed in Pubmed: 11920587.

11. Holness CL, Simmons DL. Molecular cloning of $\mathrm{cd} 68$, a human macrophage marker related to lysosomal glycoproteins. Blood. 1993; 81(6): 1607-1613.

12. Klüver H, Barrera E. A Method for the Combined Staining of Cells and Fibers in the Nervous System. J Neuropathol Exp Neurol. 1953; 12(4): 400-403, doi: 10.1097/00005072195312040-00008.

13. Lee $Y$, McKinnon PJ. Detection of apoptosis in the central nervous system. Methods Mol Biol. 2009; 559: 273-282, doi: 10.1007/978-1-60327-017-5_19, indexed in Pubmed: 19609763

14. Leins A, Riva $P$, Lindstedt $R$, et al. Expression of tenascin- $C$ in various human brain tumors and its relevance for survival in patients with astrocytoma. Cancer. 2003; 98(11): 2430-2439, doi: 10.1002/cncr.11796, indexed in Pubmed: 14635078.

15. Machaalani R, Radford JL, Waters KA. Tissue fixation effects on immunohistochemical staining of caspase- 3 in brain tissue. Appl Immunohistochem Mol Morphol. 2007; 15(4): 463-470, doi: 10.1097/01. pai.0000213150.56687.78, indexed in Pubmed: 18091392.

16. Mason JT, O'Leary TJ. Effects of formaldehyde fixation on protein secondary structure: a calorimetric and infrared spectroscopic investigation. J Histochem Cytochem. 1991; 39(2): 225-229, doi: 10.1177/39.2.1987266, indexed in Pubmed: 1987266.

17. Midwood KS, Orend G. The role of tenascin-C in tissue injury and tumorigenesis. J Cell Commun Signal. 2009; 3(34): 287-310, doi: 10.1007/s12079-009-0075-1, indexed in Pubmed: 19838819.
18. Midwood KS, Hussenet $T$, Langlois B, et al. Advances in tenascin-C biology. Cell Mol Life Sci. 2011; 68(19): 3175-3199, doi: 10.1007/s00018-011-0783-6, indexed in Pubmed: 21818551.

19. Pikkarainen M, Martikainen $P$, Alafuzoff I. The effect of prolonged fixation time on immunohistochemical staining of common neurodegenerative disease markers. J Neuropathol Exp Neurol. 2010; 69(1): 40-52, doi: 10.1097/ NEN.0b013e3181c6c13d, indexed in Pubmed: 20010304.

20. Ploeger S, Guldemond JM, Feirabend HK, et al. Acidification of human brains stored in fixatives. Eur J Morphol. 1993; 31 (4): 286-290.

21. Porter AG, Jänicke RU. Emerging roles of caspase-3 in apoptosis. Cell Death Differ. 1999; 6(2): 99-104, doi: 10.1038/ sj.cdd.4400476, indexed in Pubmed: 10200555.

22. Sheaffer S, Rosoklija G, Dwork AJ. Myelin staining of archival brain tissue. Clin Neuropathol. 1999; 18(6): 313-317.

23. Smith EE. Cerebral amyloid angiopathy as a cause of neurodegeneration. J Neurochem. 2018; 144(5): 651-658, doi: 10.1111/jnc.14157, indexed in Pubmed: 28833176.

24. Stankov A, Belakaposka-Srpanova V, Bitoljanu N, et al. Visualisation of Microglia with the use of Immunohistochemical Double Staining Method for CD-68 and Iba-1 of Cerebral Tissue Samples in Cases of Brain Contusions. Pril (Makedon Akad Nauk Umet Odd Med Nauki). 2015; 36(2): 141-145, doi: 10.1515/prilozi-2015-0062, indexed in Pubmed: 27442380.

25. Sun $Y, X u Y$, Geng L. Caspase-3 inhibitor prevents the apoptosis of brain tissue in rats with acute cerebral infarction. Exp Ther Med. 2015; 10(1): 133-138, doi: 10.3892/ etm.2015.2462, indexed in Pubmed: 26170924.

26. Walker DG, Lue LF. Immune phenotypes of microglia in human neurodegenerative disease: challenges to detecting microglial polarization in human brains. Alzheimers Res Ther. 2015; 7(1): 56, doi: 10.1186/s13195-015-0139-9, indexed in Pubmed: 26286145.

27. Webster JD, Miller MA, Dusold D, et al. Effects of prolonged formalin fixation on diagnostic immunohistochemistry in domestic animals. J Histochem Cytochem. 2009; 57(8): 753-761, doi: 10.1369/jhc.2009.953877, indexed in Pubmed: 19398606.

28. Weickenmeier J, de Rooij R, Budday S, et al. Brain stiffness increases with myelin content. Acta Biomater. 2016; 42: 265-272, doi: 10.1016/j.actbio.2016.07.040, indexed in Pubmed: 27475531.

29. Wiese S, Karus M, Faissner A. Astrocytes as a source for extracellular matrix molecules and cytokines. Front Pharmacol. 2012; 3: 120, doi: 10.3389/fphar.2012.00120, indexed in Pubmed: 22740833.

30. Wilcock DM, Gordon MN. Quantification of cerebral amyloid angiopathy and parenchymal amyloid plaques with congo red histochemical stain. Nat Protoc. 2006; 1(3): 1591-1595.

31. Williams JH, Mepham BL, Wright DH. Tissue preparation for immunocytochemistry. J Clin Pathol. 1997; 50(5): 422-428, doi: 10.1136/jcp.50.5.422. 